\title{
PROMOCI ÓN DE LA SALUD Y UN ENTORNO LABORAL SALUDABLE
}

\author{
Sara Barrios Casas $^{1}$ \\ Tatiana Paravic Klijn²
}

Casas SB, Klijn TP. Pde la salud y su entorno laboral saludable. Rev Latino-am Enfermagem 2006 janeirofevereiro; 14(1): 136-41.

En este estudio se exponen abordajes sobre un entorno laboral saludable, presentándose las principales líneas en esta temática. Es en este sentido que un entorno laboral saludable promueve una buena salud, que es un recurso primordial para el desarrollo social, económico y personal, así como una importante dimensión de los ambientes laborales. Es por esta razón que, para que exista un entorno laboral saludable, se debe promover la participación de todos los actores para controlar, mejorar y mantener la salud y el bienestar de los trabajadores $\mathrm{y}$, así, propender a un ambiente laboral saludable, donde se mejore la calidad de vida de toda la población, ya que este es uno de los bienes más preciados por las personas, comunidades y países. Es en este ámbito que los profesionales de enfermería, a través de la promoción en salud, van a promover entornos laborales saludables, fomentando el desarrollo personal, familiar y social, apoyando de esta manera al cumplimiento de las metas definidas por empleadores y trabajadores.

DESCRIPTORES: trabajo; ambiente de trabajo; promoción de la salud

\section{HEALTH PROMOTI ON AND A HEALTHY WORKPLACE}

This study discusses approaches to a healthy working environment and presents the main lines in this theme area. A healthy workplace promotes good health, which is a basic resource for social, economic and personal development, as well as an important aspect of the working environment. The creation of a healthy workplace depends on promoting the participation of all parties involved, in order to monitor, improve and maintain employees' health and well-being, moving towards a healthy work environment that will improve the quality of life of the population in general, since good health is one of the most valued conditions by individuals, communities and countries. In this context, nursing professionals can encourage healthy workplaces by promoting good health, thus assisting in the development of individuals, families and society and, in this way, supporting the achievement of the goals set by employers and employees.

DESCRIPTORS: work; working environment; health promotion

\section{PROMOÇÃO DE SAÚDE E O LUGAR DE TRABALHO SAUDÁVEL}

Este estudo expõe abordagens a um ambiente de trabalho saudável, apresentando as principais linhas nesta temática. Um lugar de trabalho saudável promove boa saúde, considerado um recurso básico para o desenvolvimento social, econômico e pessoal, e também um aspecto importante do ambiente de trabalho. Para que exista um lugar de trabalho saudável, deve-se promover a participação de todos os envolvidos, com vistas a monitorar, melhorar e manter a saúde e o bem-estar dos trabalhadores e, assim, mover em direção a um ambiente de trabalho saudável, melhorando a qualidade de vida da população em geral, já que uma boa saúde é um dos bens mais valorizados pelos indivíduos, comunidades e países. Nesse contexto, enfermeiros profissionais podem, a través da promoção da saúde, promover ambientes de trabalho saudáveis, estimulando o desenvolvimento pessoal, familiar e social e, assim, apoiando o alcance das metas fixadas por empregadores e empregados.

\footnotetext{
${ }^{1}$ Enfermera. Magíster en Educación. Profesor Asistente Depto. de Pediatría y Cirugía Infantil. Facultad de Medicina. Universidad de La Frontera. Temuco. Chile. Alumna Doctorado en Enfermería. Universidad de Concepción. Chile. e-mail: sbarrios@ufro.cl; ${ }^{2}$ Enfermera. Magíster en Enfermería en Salud Comunitaria. Universidad de Concepción. Doctora en Enfermería. Universidad de Sao Paulo. RP. Brasil. Profesor Titular Depto. de Enfermería. Facultad de Medicina. Universidad de Concepción. Chile. e-mail: tparavic@udec.cl
} 


\section{NTRODUCCIÓN}

La Organización Panamericana de la Salud (OPS) considera al lugar de trabajo como un entorno prioritario para la promoción de la salud en el siglo $X X I$. La salud en el trabajo y los entornos laborales saludables se cuentan entre los bienes más preciados de personas, comunidades y países. Un entorno laboral saludable es esencial, no sólo para lograr la salud de los trabajadores, sino también para hacer un aporte positivo a la productividad, la motivación laboral, el espíritu de trabajo, la satisfacción en el trabajo y la calidad de vida general ${ }^{(1)}$.

Un lugar de trabajo saludable promueve una buena salud, que es un recurso primordial para el desarrollo social, económico y personal, así como una importante dimensión de los ambientes laborales. Diversos factores como los políticos, económicos, sociales, culturales, ambientales y biológicos pueden favorecer o dañar la salud de los trabajadores pero, si el entorno laboral es saludable, se habrá adelantado en la conquista de espacios que permitan el desarrollo y promoción de la salud en el trabajo.

En este texto, se revisa como, a través de la promoción en salud, se pueden desarrollar entornos laborales saludables, ya que el lugar de trabajo es donde las personas pasan la tercera parte de sus vidas y es por eso la importancia de impulsar ambientes laborales saludables. En este sentido, la OPS ha considerado al lugar de trabajo como un entorno prioritario para la promoción de la salud en el siglo $X X X^{(1)}$, debido a la importancia que ha tomado la fuerza laboral y la incidencia económica que tiene en los niveles de producción. Todo esto posibilita que se requiera abordar esta temática en profundidad.

Fueron utilizadas, en este estudio, publicaciones de los últimos diez años de autores exponentes en el tema.

\section{ENTORNO LABORAL SALUDABLE}

Si tenemos en cuenta que la salud no es sólo la ausencia de enfermedad; y que se debe atender en su preservación y promoción, no sólo a los aspectos físicos, sino también a los psíquicos y sociales; la salud puede considerarse en términos de capacidad y posibilidad de satisfacer necesidades vitales, entre las cuales incluimos la autonomía, la alegría y la solidaridad. La salud también implica lucha y negociación con el medio ambiente, tanto a nivel individual como colectivo, y guarda relación con las condiciones de trabajo. Una manera de lograr los estados anteriormente descritos es a través del proceso de capacitación de las personas para aumentar el control sobre su salud y mejorarla, descrito por la Organización Mundial de la Salud ${ }^{(2)}$ como "promoción de la salud". En la conferencia de Ottawa en $1986^{(3-4)}$, se planteó que la promoción en salud implica a los procesos que favorecen el control de los individuos y grupos sobre las variables que condicionan su propia salud.

Esta se constituye entonces en un proceso político y social global que abarca no solamente las acciones dirigidas a fortalecer las habilidades y capacidades de los individuos, sino también las dirigidas a modificar las condiciones sociales, ambientales y económicas ${ }^{(5-6)}$. Esta promoción en salud debe ser extensible hacia los lugares de trabajo de las personas, quienes pasan la tercera parte de sus vidas en esta actividad.

Para la OMS y la OPS ${ }^{(1)}$, la promoción de la salud en el lugar de trabajo incluye la realización de una serie de políticas y actividades en los lugares de trabajo, diseñadas para ayudar a los empleadores y trabajadores en todos los niveles a aumentar el control sobre su salud y a mejorarla, favoreciendo la productividad y competitividad de las empresas y contribuyendo al desarrollo económico y social de los países. El lugar de trabajo puede ser cualquier entorno en el cual la gente trabaja, incluidos el hogar y la calle. La promoción de la salud en el lugar de trabajo convoca la participación de trabajadores, empleadores y otros actores sociales interesados en la puesta en práctica de iniciativas acordadas en forma conjunta para la salud y el bienestar de la fuerza laboral(7-8), para lo cual se generan principios fundamentales, en cuanto a la promoción de la salud en el lugar de trabajo(1), que se describen a continuación:

1. Carácter participativo y empoderador. Se promueve la participación de los trabajadores y directivos, a menudo a través de la organización de comités de salud, seguridad e higiene ocupacional. La participación en las decisiones que afectan su salud brinda una mayor seguridad a los trabajadores, en su capacidad para hacer cambios en su vida y desarrollar habilidades para la promoción y protección de la salud. Además, permite que ellos puedan tener y desarrollar sus propias iniciativas en este campo. 
2. Cooperación multisectorial y multidisciplinaria. Participación de todos los actores sociales interesados de los diferentes sectores, tales como el gobierno, las empresas y lugares de trabajo, los sindicatos, el sector de la salud, las instituciones de enseñanza superior, las organizaciones no gubernamentales, la comunidad y otras entidades.

3. Justicia social. Los programas se ofrecen a todos los miembros del lugar de trabajo, independientemente de su cargo, tipo de contrato, nacionalidad, sexo o grupo étnico.

4. Sostenibilidad. Para lograr este principio, la promoción y la protección de la salud en los lugares de trabajo deben convertirse en parte integral de la gestión y organización de la empresa o lugar de trabajo y de la organización comunitaria que la rodea. 5. Carácter integral. Los programas reconocen el efecto combinado de los factores personales, ambientales, organizacionales, comunitarios, sociales e informativos sobre el bienestar del trabajador.

Utilizando como marco de referencia la Carta de Ottawa de la $\mathrm{OMS}^{(1)}$, sobre la Promoción de la Salud, la misma OMS adaptó ésta al contexto de salud y trabajo. Por tanto, las intervenciones correspondientes a la promoción de la salud en el lugar de trabajo pueden incluir actividades en las siguientes áreas, entre otras:

- Construir políticas públicas de trabajo saludable para todos los sectores de la vida productiva internacional, nacional y local: definiciones políticas y operativas por parte de todos los actores sociales interesados en fomentar, promover y proteger la salud de los trabajadores, mediante la expedición de normas, reglamentos, planes y programas que conduzcan a ello.

- Fortalecimiento de la organización y participación de la comunidad trabajadora y general, a través de los comités o comisiones de salud y seguridad conjuntas entre empleadores y trabajadores, y de la acción comunitaria a nivel intersectorial, en materia de condiciones del ambiente general, de trabajo, de vivienda, de educación y de vida, entre otros.

- Desarrollo de habilidades y responsabilidades personales y colectivas, relacionadas con la gestión de la salud, la seguridad, el autocuidado y el desarrollo personal de los trabajadores, sus organizaciones y las comunidades a su alrededor para proteger y mejorar la salud: fundamentados en estilos de trabajo y de vida saludables en la búsqueda de mejores condiciones y calidad de vida laboral, personal, familiar y comunitaria, tales como la capacitación sobre los factores de riesgo en el ambiente físico, los métodos para protegerse y fomentar comportamientos saludables en el trabajador, como son el abandono del hábito de fumar, una mejor alimentación y la práctica periódica de ejercicios físicos.

- Reorientar los servicios de salud ocupacional y otros servicios de salud, para incluir la promoción de la salud y todos sus aspectos relacionados dentro de sus agendas y lograr un mayor acceso del trabajador a los servicios de salud primaria, preventiva y ocupacional. Pretende trascender el horizonte clínico del servicio para buscar las soluciones en materia de Promoción de la Salud de los Trabajadores y de prevención de la enfermedades, encaminados a la mejor protección de su salud y de sus grupos familiares.

- Crear ambientes favorables en el sitio de trabajo, partiendo del concepto integral del puesto de trabajo, sin admitir barreras en su alcance. Se debe incluir la clara identificación de las condiciones y medio ambiente de trabajo, los procesos productivos y la identificación de necesidades de los trabajadores, así como del ambiente general y las poblaciones circunvecinas a la empresa, que permitan orientar las soluciones para el adecuado control de los riesgos del trabajo, realizando acciones tales como modificaciones para eliminar los factores de riesgo para la salud y la seguridad en el entorno físico, cambios en la forma de organizar el trabajo, etc.

Los elementos enunciados dependerán de niveles de relación, características del ambiente laboral o bien estrategias que potencien la promoción de la salud. Los principales aspectos a considerar son los siguientes: la relación estrecha entre la salud de los trabajadores y la productividad, la motivación laboral, la satisfacción en el trabajo y la calidad de vida en general. El lugar de trabajo es el sitio clave para desarrollar estrategias de promoción de la salud porque es el lugar en el que pasamos gran parte del día junto a personas de diferentes procedencias, etnia, sexo y formación. Es en este contexto que el entorno laboral es un fenómeno complejo y que ni las reformas, ni el desempleo son temas ajenos al de la calidad de vida en el trabajo porque, directa o indirectamente, son elementos interrelacionados ${ }^{(9)}$. Los lugares de trabajo han cambiado considerablemente en las últimas décadas, no solamente con la automatización, sino también con el incremento de trabajos y modalidades diferentes 
(por ejemplo, trabajo a contrato fijo, honorario, o las posibilidades de trabajar en casa o independiente). Estos cambios, con aquellos que se están viviendo con la globalización, como son las altas tasas de desempleo y la reducción de las planillas de las empresas, contribuyen a la creación de un ambiente laboral muy complejo, el cual tiene un efecto e impacta sobre la salud de los trabajadores ${ }^{(10)}$. Se debe colocar el acento en el factor humano o si se quiere en la humanización de los ambientes laborales y esto requiere de un cambio cultural de envergadura ${ }^{(9)}$.

Es así que el entorno laboral va a ser el conjunto de cualidades, atributos o propiedades relativamente permanentes de un ambiente de trabajo concreto que son percibidas, sentidas o experimentadas por las personas que componen la organización empresarial y que influyen sobre su conducta, la satisfacción y la productividad. Está relacionado con el «saber hacer» del directivo, con los comportamientos de las personas, con su manera de trabajar y de relacionarse, con su interacción con la empresa, con las máquinas que se utilizan y con la propia actividad de cada uno(11-12)

Un entorno laboral saludable tiene componentes objetivos y subjetivos. Dicho en términos simples, es un indicador de satisfacción con la manera cómo las personas viven la cotidianeidad en su ámbito laboral ${ }^{(13-14)}$. Involucra desde la situación laboral objetiva, es decir, las condiciones de trabajo en un sentido amplio, tanto las condiciones físicas como las contractuales y remuneraciones, hasta las relaciones sociales que se dan tanto entre los trabajadores como entre éstos y la parte empresarial. Entre los componentes subjetivos tenemos las actitudes y los valores de los sujetos y las percepciones de satisfacción o insatisfacción que derivan de esta conjunción de factores ${ }^{(9)}$. Esta subjetividad y la suma de variables objetivas existentes determinarán las respuestas que darán las personas cuando son consultadas por aspectos de su trabajo. Sus respuestas tienen que ver con la percepción sobre estas variables, que es el resultado de una ecuación personal sobre estas características subjetivas y objetivas del trabajo. Las personas trabajan para satisfacer necesidades económicas, pero también de desarrollo personal. Estas necesidades dan lugar a las motivaciones que facilitan el rendimiento, por lo que la percepción está determinada por la historia del sujeto y de sus anhelos y proyectos personales.

Es en este contexto que el entorno laboral saludable son aquellos centros de trabajo en los que las condiciones van dirigidas a lograr el bienestar de los trabajadores pero no sólo en el sentido de un buen ambiente físico, se trata además de que existan buenas relaciones personales, buena organización, salud emocional, y que se promueva el bienestar familiar y social de los trabajadores a través de la protección de riesgos, estimulando su autoestima y el control de su propia salud y del ambiente laboral. Todos estos factores están interrelacionados dinámicamente.

Dentro del ámbito laboral, el entorno físico del lugar de trabajo va a impactar directamente en la salud y seguridad de los trabajadores, como lo son los puestos de trabajo, las características ambientales como el frío, calor, ruido e iluminación.

El entorno psico-social que hoy día es una de las características del modelo económico abierto, orientado hacia el mercado externo y con economías interrelacionadas, tiene un componente de incertidumbre que deviene justamente de la imposibilidad de controlar todos los factores y particularmente el comportamiento de la demanda ${ }^{(9)}$. Esto se traduciría en un temor frente a la inestabilidad del empleo, ya que el principal factor de ajuste es el despido y en ese escenario el desafío que se plantea es flexibilizar la organización de las empresas. La pérdida del empleo, o el miedo a perderlo, se constituyen así en un marco de inseguridad, que afecta el colectivo laboral y que genera fuertes presiones sobre las condiciones psicosociales y sobre las condiciones de trabajo, generando cuadros de estrés, riesgos en la salud física y mental y crecientes grados de insatisfacción laboral, que inciden en un ambiente laboral no saludable. Al respecto, en 1998 el PNUD reveló que estudios realizados por esa institución mostraban importantes señales de inseguridad, descontento y otros efectos psicosociales sobre la población chilena. Al momento de evaluar la confianza en mantener el empleo, casi el $40 \%$ emitió una evaluación negativa, proporción que se elevaba a casi el $70 \%$ en la posibilidad de encontrar un trabajo aceptable en caso de perder el empleo actual ${ }^{(15)}$. La 
estabilidad laboral constituye por tanto un indicador para valorar el ambiente laboral saludable(16). El entorno social, como el manejo organizacional, las normas y los procedimientos, la organización del trabajo, el control que los trabajadores tienen sobre el trabajo, la comunicación efectiva, la cohesión de grupos, la carga de trabajo y la participación de los trabajadores en la toma de decisiones ${ }^{(10)}$ contribuyen también a la salud y bienestar de los trabajadores. Las respuestas del trabajador a su entorno físico y psicosocial, dependerán de factores individuales y de su predisposición genética.

Enfermería puede tener una labor destacada en la generación de entornos laborales saludables. EI profesional de enfermería que se desenvuelva en salud laboral debe tener presente ciertas estrategias, como el desarrollo de destrezas y habilidades, que consiste en apoyar a trabajadores en la elección de alternativas saludables a través del uso de procesos educativos que aumenten o cambien sus conocimientos, actitudes, intenciones y motivaciones $^{(10,17)} y$, así, facilitar, apoyar y fomentar el desarrollo de hábitos saludables para los trabajadores y su grupo familiar, que incluye la creación de oportunidades para generar, mantener y reforzar cambios de comportamiento y remover los obstáculos que interfieren con ellos, a través de la participación activa de todos los actores importantes: administradores, empresarios y trabajadores.

\section{REFERENCI AS BI BLI OGRÁFI CAS}

1. OMS, OPS. Estrategia de Promoción de la salud en los lugares de trabajo de América Latina y el Caribe: Anexo № 6 - Documento de Trabajo. Ginebra. (Swz): Organización Mundial de la Salud; 2000.

2. Solé M. La promoción de salud en el trabajo: ¿utopía o necesidad?. Centro Nacional de Condiciones de Trabajo. Argentina. [publicación periódica en línea] 2004; [citada 2004 septiembre 20]. Disponible en: http:// www.estrucplan.com.ar

3. OMS. Declaración de Ottawa. Conferencia Internacional sobre Promoción de Salud. Ottawa. (Canadá): Organización Mundial de la Salud; 1986.

4. Alvarez-Dardet C. Promoción de la salud. Departamento de Salud Pública. Universidad de Alicante. España. [publicación periódica en línea] 2002; [citada 2004 septiembre 20]. Disponible en: http://perso.wanadoo.es 5. Consejo Nacional para la Promoción de Salud Vida Chile. Plan Estratégico 2001-2006. Metas intersectoriales al 2006. Santiago. Chile: Ministerio de Salud; 2000.

\section{CONCLUSIÓN}

El entorno laboral saludable constituye un ámbito prioritario para la promoción de la salud en el siglo XXI. Este constituye un bien que potenciará el desarrollo de personas, comunidades y países, constituyéndose en un elemento central de la calidad de vida general.

Para que exista un entorno laboral saludable, éste debe tener las siguientes características: promover la participación de los empleadores, trabajadores y otros actores sociales interesados en la realización de acciones conjuntas para controlar, mejorar y mantener la salud y el bienestar de los trabajadores; y la realización de procesos orientados a lograr el empoderamiento de empleados y empleadores. Un ambiente laboral saludable asegura la salud de los trabajadores y mejora las condiciones de la productividad, por lo tanto, la calidad de vida de toda la población.

Los profesionales de enfermería, a través de la promoción en salud promueven entornos laborales saludables, fomentando el desarrollo personal, familiar y social, apoyando de esta manera al cumplimiento de las metas definidas por empleadores y trabajadores, logrando de esta manera centros de trabajo con condiciones dirigidas hacia el bienestar de los trabajadores, que fomenten las buenas relaciones personales, una buena organización y buena salud emocional.

6. Consejo Nacional para la Promoción de Salud Vida Chile. Cuenta Pública 2001. Santiago (Chile): Ministerio de Salud; 2002 .

7. CIE. El CIE y la movilización de las enfermeras para la promoción de la salud. [publicación periódica en línea] 2000; [citada 2004 septiembre 20]. Disponible en: http:// www.icn.ch

8. Ministerio de Salud (Chile). Planes Comunales de Promoción de la Salud. Documento de trabajo. Santiago (Chile): Ministerio de Salud; 2002.

9. Espinoza M. Calidad de Vida en el Trabajo: Reflexiones en torno a la Inseguridad y el Malestar Social: Temas Laborales № 18. Santiago. Chile: Ministerio del Trabajo; 2001.

10. Salamo P. Ambiente Laboral y centros de Salud. Diplomado en Promoción de la Salud. Instituto de Nutrición y Tecnología de los Alimentos. Santiago. (Chile): Universidad de Chile; 2002 .

11. Universidad de Champagnt. Clima Laboral. [publicación periódica en línea] 2004; [citada 2004 septiembre 18]. Disponible en: http://www.gestiopolis.com/canales/derrhh/ articulos/42/clima.htm 
12. Gestiopolis. Clima Laboral. [publicación periódica en línea] 2004; [citada 2004 septiembre 18]. Disponible en: http:// www.gestiopolis.com/dirgp/rec/clima.htm

13. Salinas J, Vio F. Promoción de la Salud en Chile. Rev, Chil, Nutr, 2002; 29 (4): 164-73.

14. Restrepo H, Malaga H. Promoción de la Salud: como construir vida saludable. Bogotá (Co): Ed. Médica Internacional; 2001.

15. Valdés T, Gomáriz E. Mujeres Latinoamericanas en cifras: Avance de Investigación. Documento de Trabajo № 5: Santiago. Chile: Servicio Nacional de la Mujer; 1994.

16. Benavides F, Ruiz-Frutos C, García A. Salud Laboral. Conceptos y técnicas para la prevención de riesgos laborales. 2a ed. Barcelona. (Es): Editorial Masson; 2000.

17. Merino B, Arroyo H, Silva J. La evidencia de la Eficacia de la Promoción de la Salud. Configurando la Salud Pública en una Nueva Europa. Informe de la Unión Internacional de Promoción de la Salud y Educación para la Salud para la Comisión Europea. España: Comunidad Europea; 2000. 